\title{
Interventionell-radiologische Therapien in der pneumologischen Onkologie
}

\author{
Interventional Radiological Therapies in Lung Oncology
}

Autoren

Institute
D. Frechen ${ }^{1}$, S. Krüger ${ }^{1}$, C. Cornelissen ${ }^{1}$, P. Bruners ${ }^{2}$, A. H. Mahnken ${ }^{2}$

Medizinische Klinik I, Klinik für Kardiologie, Pneumologie und Angiologie, Universitätsklinikum der RWTH Aachen (Direktor: Univ.-Prof. Dr. med. N. Marx)

Klinik für Diagnostische und Interventionelle Radiologie, Universitätsklinikum der RWTH Aachen

(Direktorin: Univ.-Prof. Dr. med. C. Kuhl) eingereicht 11.1.2011

akzeptiert 28.4.2011

\section{Bibliografie}

Dol http://dx.doi.org/ 10.1055/s-0030-1256503

Online-Publikation: 1. 7. 2011

Pneumologie 2011; 65:

525-531 @ Georg Thieme

Verlag KG Stuttgart · New York

ISSN 0934-8387

\section{Korrespondenzadresse}

Dr. med. Dirk Frechen

Medizinische Klinik I

Universitätsklinikum der RWTH

Aachen

Pauwelsstr. 30

52057 Aachen

dfrechen@ukaachen.de

\section{Zusammenfassung \\ $\nabla$}

Interventionell-radiologische Verfahren zur Behandlung von primären und sekundären pulmonalen Malignomen gewinnen zunehmend an Bedeutung. Neben thermisch abladierenden Verfahren ermöglicht die selektive Instillation von Zytostatika und Embolisaten über vaskuläre Zugänge eine lokal begrenzte Therapie. Die Therapien kommen für Patienten, bei denen ein reduzierter Allgemeinzustand keine systemische Chemotherapie zulässt, palliativ in Betracht. Bei funktionell inoperablen Patienten sind insbesondere die ablativen Verfahren eine potenziell kurative Alternative zur Operation. Dieser Artikel soll eine Übersicht über die derzeit angewandten interventionell-radiologischen Verfahren in der pneumologischen Onkologie und ihren Stellenwert geben. Ob die aufgrund ihres günstigen Risiko-Nutzen-Verhältnisses vielversprechenden interventionell-radiologischen Verfahren möglicherweise eine Alternative zur strahlentherapeutischen Behandlung darstellen oder in multimodalen Konzepten überlegen sind, müssen Studien zeigen.

\section{Einleitung}

\section{$\nabla$}

Pulmonale Rundherde sind sehr unterschiedlicher Genese. Neben lokalisierten entzündlichen oder gutartigen Erkrankungen wie zum Beispiel Fibromen oder Hamartomen treten sie bei verschiedenen Granulomatosen im Rahmen einer Systemerkrankung auf. Meist handelt es sich jedoch um primär pulmonale Malignome oder Metastasen eines extrapulmonalen Primarius.

Für Patienten mit nicht-kleinzelligem Bronchialkarzinom (NSCLC) in einem prinzipiell kurativ operablen Tumorstadium, die aufgrund ihrer eingeschränkten Lungenfunktion oder Komorbidität inoperabel sind, wird eine strahlentherapeutische Behandlung in der S3-Leitlinie zur Prävention, Diagnostik, Therapie und Nachsorge des

\section{Abstract \\ $\nabla$}

Interventional radiological procedures for the treatment of primary and secondary pulmonary malignancies have become increasingly important. In addition to thermally ablative treatment, selective chemoembolisation by a vascular access allows localised therapy. These treatments are considered to be palliative for patients in a reduced general condition which does not allow systemic chemotherapy. In functionally inoperable patients especially the ablative procedures are potentially curative alternatives to surgery. This article provides an overview of the currently used interventional radiological procedures in lung oncology and assesses their importance. Further studies are needed to show whether interventional radiological procedures, which are promising due to their favourable risk-benefit ratio, may represent an alternative to radiotherapy or be effective in multimodal approaches.

Lungenkarzinoms empfohlen [1]. Trotz prinzipiell möglicher kurativer Operabilität des Lungenkarzinoms kann allerdings in mehr als 20\% der Fälle den Patienten aufgrund ihrer Komorbidität keine Operation angeboten werden [2]. Insbesondere für diese Patienten rücken neben strahlentherapeutischen Ansätzen alternative, potentiell kurative Verfahren in den Fokus.

Für Patienten mit isolierten pulmonalen Metastasen konnte auf Basis der Daten eines internationalen Registers gezeigt werden, dass eine vollständige chirurgische pulmonale Metastasektomie potentiell kurativ sein kann [3]. Unter derselben Rationale wurden verschiedene interventionell-radiologische Verfahren für funktionell oder aufgrund ihrer Komorbidität inoperable Patienten als Alternative zum chirurgischen Vorgehen 


\begin{tabular}{|c|c|c|c|}
\hline perkutaner Zugangsweg & transpulmonal & transvenös & transarteriell \\
\hline \multirow[t]{4}{*}{ Verfahren } & Radiofrequenzablation & $\begin{array}{l}\text { transpulmonale } \\
\text { Chemoembolisation }\end{array}$ & $\begin{array}{l}\text { multiarterielle } \\
\text { Chemoperfusion }\end{array}$ \\
\hline & Mikrowellenablation & & \\
\hline & Kryoablation & & \\
\hline & Laserablation & & \\
\hline Therapieareal & Tumor & $\begin{array}{l}\text { tumorbefallenes } \\
\text { Lungensegment }\end{array}$ & $\begin{array}{l}\text { Tumor über seine arte- } \\
\text { rielle Blutversorgung }\end{array}$ \\
\hline Therapieziel & $\begin{array}{l}\text { thermische Ablation } \\
\text { des Tumorgewebes }\end{array}$ & Tumorverkleinerung & $\begin{array}{l}\text { Tumorverkleinerung, } \\
\text { evtl. Tumoreradikation }\end{array}$ \\
\hline
\end{tabular}

Tab. 1 Möglichkeiten der interventionell-radiologischen Therapien in der pneumologischen Onkologie. entwickelt. Bereits in den 1980er-Jahren veröffentlichte der Schwede Nordenström Arbeiten über den Effekt von Gleichstrom auf pulmonale Malignome. Eine Elektrode wurde dazu direkt in den Tumor eingebracht, eine andere in das Lungengewebe in einem Abstand von zwei- bis dreifachem Durchmesser des Tumors oder in die thorakale Aorta. Das Verfahren wurde als elektrochemische Behandlung bezeichnet, und je nach Polarität wurden unterschiedliche Effekte auf das Gewebe beobachtet [4]. Bis Anfang des letzten Jahrzehnts fanden interventionelle Techniken zur Tumortherapie der Lunge kaum Anwendung. Erst als interventionell-radiologische Methoden, die ursprünglich zur Behandlung von hepatischen Malignomen entwickelt wurden, zunehmend auch in der Lunge erfolgreich einsetzt wurden, hat sich die interventionell-radiologische Therapie von Lungentumoren rasch entwickelt.

Aufgrund ihres Zugangsweges lassen sich die interventionell-radiologischen Verfahren in zwei Gruppen unterteilen: Bei der einen wird perkutan eine Sonde unter Bildsteuerung transpulmonal in das Tumorgewebe vorgebracht, um das Zielgewebe anschließend mittels thermischer Energie möglichst vollständig zu destruieren. Bei der anderen Gruppe wird ein perkutaner Gefäßzugang gewählt, über den die Katheter entweder in die lungensegmentversorgenden Äste der Pulmonalarterie oder direkt in die tumorversorgenden Arterien vorgebracht werden. Eine Übersicht hierzu gibt $\bullet$ Tab. 1.

Ziel der Behandlungen ist es, lokalisiert zytotoxische Wirkungen zu erzeugen, ohne den Gesamtorganismus zu gefährden. Dies ist mittels Applikation von Hitze, Kälte, aber auch durch lokalisierte Applikation von zytotoxischen und gefäßverschließenden Substanzen möglich. Als thermoablative interventionell-radiologische Verfahren stehen die Radiofrequenzablation (RFA), die Mikrowellenablation (MWA), die Kryoablation (PCA) und die laserinduzierte Thermotherapie (LITT) zur Verfügung. Bei dem Verfahren der transpulmonalen Chemoembolisation (TPCE) wird über einen venösen Zugang eine Mischung aus Zytostatikum und Embolisat in tumorbefallene Lungensegmente instilliert. Wird wie bei der multiarteriellen Chemoperfusion ein arterieller Zugangsweg gewählt, können die tumorversorgenden arteriellen Gefäße, die häufig aus den Arteriae bronchiales abgehen, selektiv aufgesucht werden, um dort Chemotherapie zu instillieren. In diesem Artikel sollen diese derzeit für die pneumologische Onkologie verfügbaren interventionell-radiologischen Verfahren vorgestellt und bewertet werden.

\section{Perkutan-transpulmonale Verfahren \\ $\nabla$}

Bei diesen Verfahren werden perkutan-transpulmonal unter computertomografischer Kontrolle dünnkalibrige Sonden in das zu behandelnde Tumorgewebe vorgebracht. Prinzipiell kann dies unter Berücksichtigung des geplanten Eingriffs, des Allgemeinzu- stands des Patienten und seiner Komorbidität sowohl unter Analgosedierung in Kombination mit einer Lokalanästhesie als auch in Vollnarkose erfolgen. Insbesondere bei Behandlungen mit der Zielsetzung, mehrere Tumore sequenziell in einer Sitzung zu therapieren, wird aufgrund der Behandlungsdauer eine Vollnarkose bevorzugt, da hierdurch eine optimale Analgesie und Patientencompliance gewährleistet wird. Zudem erlaubt die Intubationsnarkose die wiederholte Einstellung definierter Atemlagen, was insbesondere bei komplexen Punktionswegen von Vorteil ist. Risiko und Nutzen abwägend wird dies individuell mit dem Patienten besprochen. Über die Sonden kann je nach Verfahren unterschiedlich Energie abgegeben werden, wie im Folgenden beschrieben wird. Verschiedene Sonden zur RFA und MWA sind in - Abb. 1 dargestellt. Die aktiven, Energie abgebenden Sondenspitzen sind vergrößert in $\bullet$ Abb. 2 abgebildet.

\section{Radiofrequenzablation}

Die RFA ist ein minimal-invasives hyperthermes Ablationsverfahren, welches in der Regel unter CT-Steuerung durchgeführt wird. Der Punktionsweg für die RFA-Sonde (RFS) sollte dabei so gewählt werden, dass wiederholte Passagen der Pleura vermieden werden, um das Risiko eines Pneumothorax gering zu halten. Die Abgabe eines hochfrequenten elektrischen Wechselstroms (ca. $375-480 \mathrm{kHz}$ ) über die nicht isolierte Spitze der RFS führt über die Induktion einer Ionenbewegung zur Erhitzung des Gewebes, aus der eine Proteindenaturierung resultiert. Hierbei wird für etwa 15 min eine Temperatur von bis $\mathrm{zu} 95^{\circ} \mathrm{C}$ erreicht und das Gewebe hierdurch „verkocht“. Die Ablation wird beendet, wenn die Temperatur oder der Gewebewiderstand an der Sondenspitze einen bestimmten Grenzwert überschreitet. Durch das einmalige Einbringen einer Ablationssonde können derzeit Tumoren mit einem maximalen Durchmesser von etwa $3,5 \mathrm{~cm}$ in kurativer Intention behandelt werden, um einen ausreichenden Sicherheitsabstand im Gesunden zu erhalten. Bei größeren Tumoren wird die Sonde falls erforderlich mehrfach repositioniert, um den Gesamttumor durch mehrere überlappende Behandlungsvolumina zu erfassen. Hierbei erhöht sich das Komplikationsrisiko deutlich [5]. Bei Tumoren $>3,5 \mathrm{~cm}$ gelingt eine vollständige Tumorablation signifikant seltener als bei kleineren Tumoren [6]. Nach Abschluss der Ablation kann die Sonde aktiviert zurückgezogen werden, um den intrapulmonalen Zugangsweg zu koagulieren. Hierdurch sollen ein Verschleppen von Tumorzellen und eine Blutung im Stichkanal verhindert werden. Bei einer Sitzung können sequenziell mehrere, bisher bis zu drei, Tumore, die sich auf der gleichen Lungenseite befinden, behandelt werden. Postinterventionell wird der Patient mindestens 4 Stunden nachbeobachtet und erhält nach 2 Stunden ein Röntgen des Thorax p.a. in Exspiration zum Ausschluss eines Pneumothorax. 


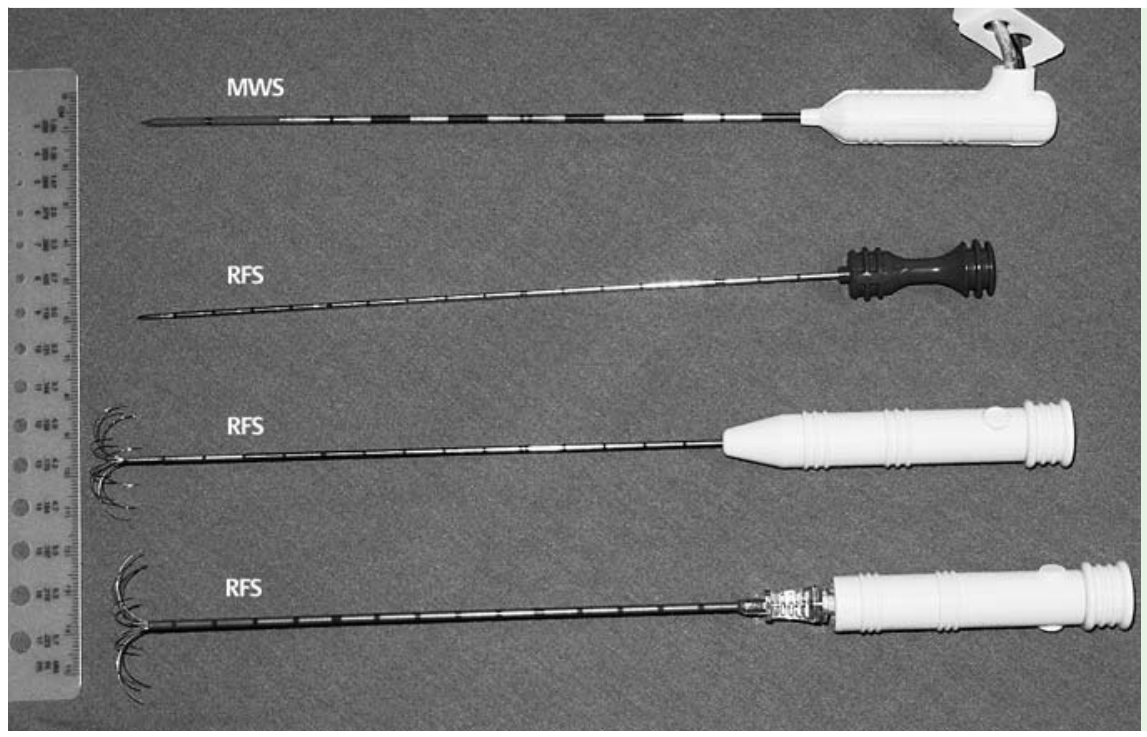

Abb. 1 Sonden in verschiedenen Größen zur Mikrowellenablation (MWS) und zur Radiofrequenzablation (RFS).

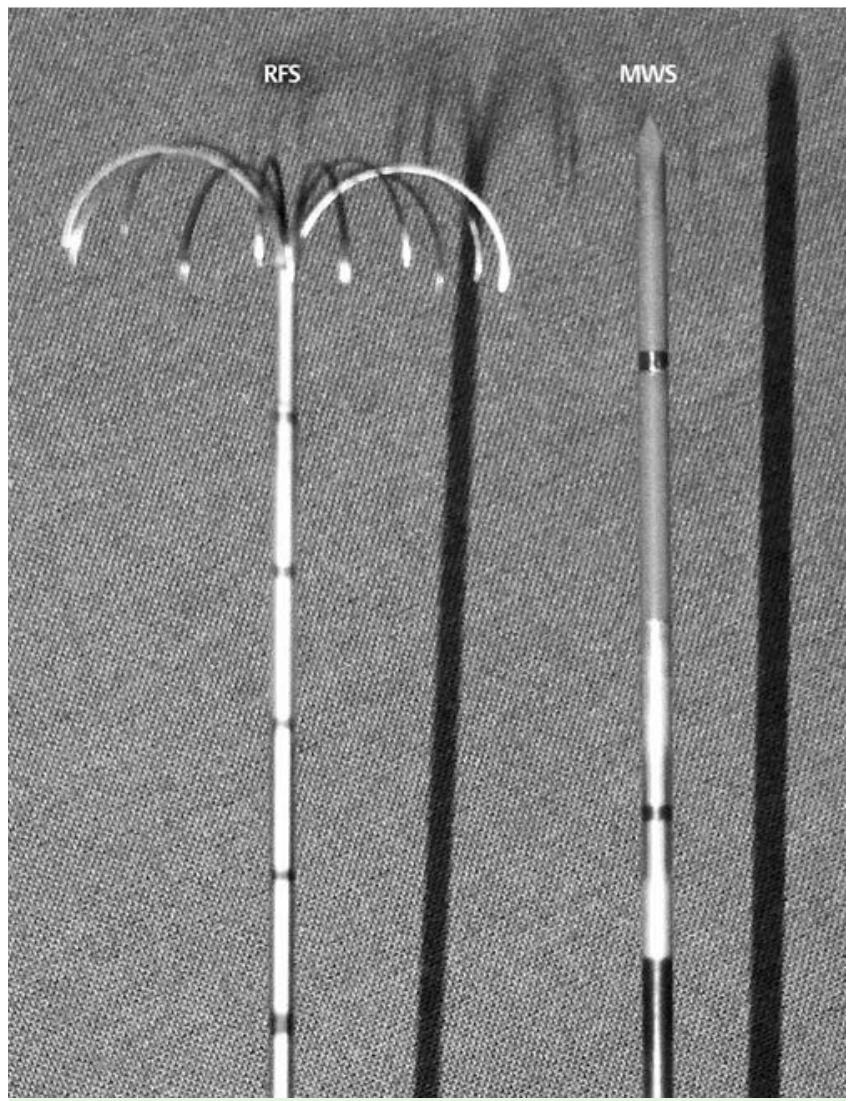

Abb. 2 Aktive, Energie abgebende Sondenspitzen zur Radiofrequenzablation (RFS) und zur Mikrowellenablation (MWS).

\section{Kontraindikationen}

Eine relative Kontraindikation stellt eine Tumornähe zu hilären Strukturen, großen Bronchien oder großen Gefäßen dar. Der Blutfluss in den Gefäßen leitet Hitze ab, sodass die Zieltemperatur in den an größere Gefäße angrenzenden Tumorbereichen nicht erreicht wird und ein hohes Rezidivrisiko besteht.

Absolute Kontraindikationen sind schwere Koagulopathien und eine Lebenserwartung $<3$ Monate.

\section{Komplikationen}

Häufigste Komplikation der pulmonalen RFA ist der Pneumothorax. Bei einem Drittel der Fälle mit interventionsbedingtem Pneumothorax ist die Behandlung mit einer einmaligen Absaugung oder einer Pleuradrainage erforderlich. Neben Einblutungen entlang des Stichkanals können hämorrhagische Pleuraergüsse bis hin zum seltenen lebensbedrohlichen Hämatothorax auftreten, der thoraxchirurgisch oder interventionell-radiologisch durch Embolisation des verursachenden Lungengefäßes behandelt werden muss. Die Behandlung pleuraständiger Tumoren birgt die Gefahr der Entstehung einer pleuralen Fistel, die häufig nur langsam abheilt und manchmal eine operative Sanierung erfordert.

Postinterventionell tritt häufig eine inflammatorische Reaktion auf, die sich systemisch durch ein erhöhtes C-reaktives Protein und einen Anstieg der Leukozytenzahl nachweisen lässt. Selten treten bakterielle Superinfektionen im Ablationsareal auf. Bei der Behandlung von 366 Lungentumoren in 211 Sitzungen bei 137 Patienten in einem Zentrum verstarb kein Patient während der Behandlung, jedoch zwei Patienten (0,9\%) im Verlauf. Ein Patient starb an einem nicht zu beherrschenden Pneumothorax und der andere an schweren Hämoptysen. Als größere Komplikationen wurden in dieser Veröffentlichung beschrieben: Pneumothorax oder Pleuraergüsse, die die Behandlung mittels Drainage erforderten, Pleuritis, Lungenabzesse und intrapulmonale Hämorrhagien mit Hämatothorax. Ihre kumulative Rate lag bei $17,1 \%[7]$.

\section{Fallbeispiel ( $\bullet$ Abb.3)}

Bei einer 67-jährigen Patientin wurde in der Nachsorge eines Mammakarzinoms eine isolierte pulmonale Metastase im rechten Lungenunterlappen diagnostiziert. Zur Intervention wurde der präinterventionelle PET-Datensatz mit den bei der Intervention angefertigten CT-Bildern überlagert. Der Tumor wurde mit einer bipolaren RFS, deren Spitze über $3 \mathrm{~cm}$ aktiv ist, abladiert. Bereits während der RFA zeigte sich das typische perifokale Infiltrat um den Tumor als Korrelat der Hitzeeinwirkung auf das den Tumor umgebende Lungenparenchym. Periinterventionell trat ein kleiner Pneumothorax auf, der sich im weiteren Verlauf selbstständig resorbierte. In einer Computertomografie einen Tag nach der Intervention stellte sich der initiale Tumor, bedingt durch eine lokale Einblutung, ein wenig größer dar. Nach 6 Mo- 

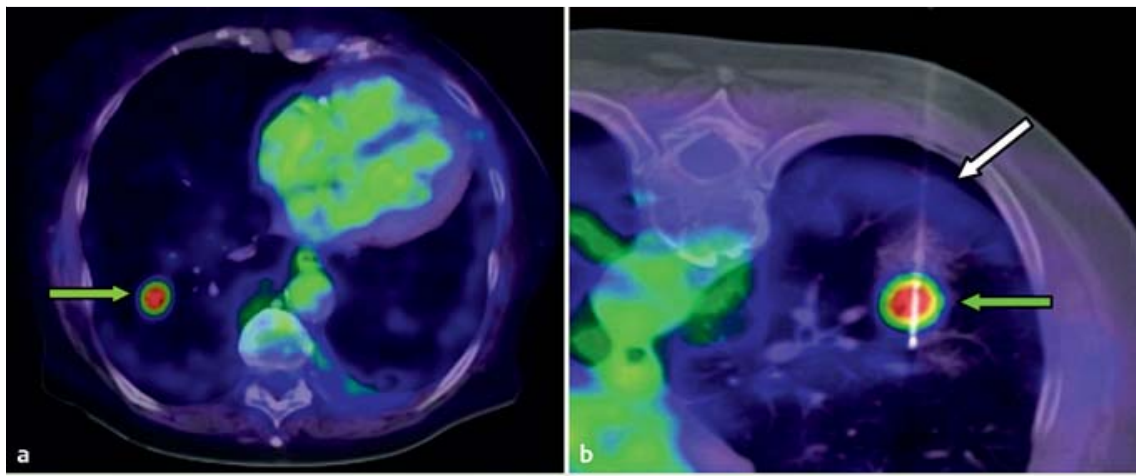

Abb. 3 Fallbeispiel Radiofrequenzablation: Grüner Pfeil: Tumor. Weißer Pfeil: Pneumothorax. a PET-positive Metastase eines Mammakarzinoms im rechten Lungenunterlappen. b Radiofrequenzablation der Metastase mit periinterventionellem Auftreten eines kleinen Pneumothorax. c 1 Tag postinterventionell. d PET-CT 6 Monate postinterventionell: kein vitaler Restbefund im Tumor, da in der PET keine FDG-Speicherung mehr nachweisbar
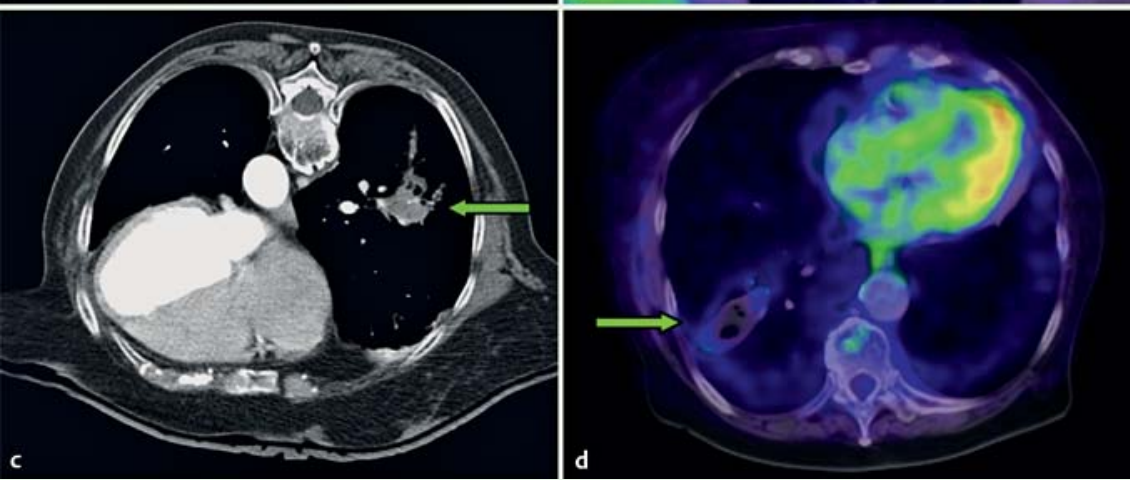
ist.

naten war der ursprüngliche Tumor im CT noch abgrenzbar, aber metabolisierte in der PET kein FDG mehr. Für die Interpretation der verlaufskontrollierenden Bilder nach RFA ist es wichtig zu wissen, dass weiterhin ein Tumor im Ablationsgebiet zu sehen ist. Dieser besteht nun aus avitalem zerstörtem Gewebe, das nicht mehr wachsen kann. Bis zu 6 Monate postinterventionell kann die Läsion durch periinterventionelles Einbluten und Ödembildung größer sein als der ursprüngliche Tumor. Im weiteren Verlauf wird das abladierte Gewebe dann narbig umgebaut und zeigt eine typische Schrumpfung. Hierbei kann je nach Größe des behandelten Volumens auch die Bildung einer Kaverne beobachtet werden.

\section{Mikrowellenablation}

Mikrowellen (900-2.500MHz) regen Wassermoleküle im Gewebe zu Schwingungen an und führen hierdurch zur Erhitzung des Gewebes. Ab einer Temperatur von ca. $60^{\circ} \mathrm{C}$ resultiert eine sofortige Proteindenaturierung. Theoretisch und im Tierexperiment ist es mit der MWA im Vergleich zur RFA möglich, in kürzerer Zeit höhere Temperaturen in einem größeren Volumen zu erzeugen [8]. Bis auf die Sonde und die über sie applizierte Energie unterscheidet sich die Behandlung nicht wesentlich von der RFA. Auch wenn nur wenige Veröffentlichungen über die Behandlung von primären und sekundären Malignomen in der Lunge vorliegen, sind unseres Erachtens Indikation, Durchführung und Komplikationen vergleichbar mit der RFA.

\section{Fallbeispiel $(\bullet$ Abb.4)}

Bei einem 60-jährigen Patienten wurde 1993 ein pulmonales Adenokarzinom im rechten Lungenoberlappen diagnostiziert. In der Ausbreitungsdiagnostik ergab sich kein Hinweis auf eine Lymphknotenfilialisierung oder auf Fernmetastasen. Bei geringer lungenfunktioneller Reserve aufgrund eines Lungenemphysems erfolgte eine atypische Resektion des Lungenoberlappens in kurativer Intention. 13 Jahre später trat ein neuer Rundherd im rechten Lungenunterlappen ohne Hinweis auf eine Filialisierung auf. Histologisch wurde ein pulmonales Adenokarzinom nachge- wiesen. In kurativer Intention erfolgte eine atypische Resektion des Unterlappens rechts. Drei Jahre später zeigte sich erneut ein Rundherd von 1,6 × 1,2 cm im Rest des rechten Lungenunterlappens mit der Histologie eines pulmonalen Adenokarzinoms. Bei nun fehlender pulmonaler Reserve mit funktioneller Inoperabilität bei einer auf 38\% vom Soll reduzierten FEV1 wurde dem Patienten eine Mikrowellenablation angeboten, in die er einwilligte. Der Eingriff wurde in potentiell kurativer Intention durchgeführt, da kein Hinweis auf eine Filialisierung bestand. In den Verlaufskontrollen mittlerweile über ein Jahr stellt sich computertomografisch ein Tumor in unveränderter Größe im Ablationsareal dar, der im PET keine FDG-Aufnahme zeigt und daher als avital anzusehen ist.

\section{Kryoablation}

Bei der perkutanen Kryoablation (PCA) wird nach Lokalanästhesie der Haut und Instillation eines Lokalanästhetikums in den Pleuraraum eine Nadel durch den zu abladierenden Tumor bis zu seinem Rand vorgebracht. Anschließend wird über die einliegende Nadel ein Dilatator mit einer Schleuse geschoben. Die Nadel und der Dilatator werden entfernt und eine Kryotherapiesonde (PCAS) bis zum Ende der Schleuse vorgebracht. Die Schleuse wird ohne die Position der PCAS in der Lunge zu verändern einige Zentimeter zurückgezogen, sodass die PCAS frei im Tumor liegt. Über die Sonde wird das Gewebe je nach Protokoll zwei- bis dreimal für 5 bis 20 Minuten eingefroren und zwischen den Zyklen aufgetaut. Die PCAS verwendet hierzu Argon und Helium. Das Einfrieren ist mehrfach erforderlich, da im normalen Lungenparenchym das initiale Einfrieren aufgrund der enthaltenen Luft nur zu einem Eisball von $1 \mathrm{~cm}$ führt. Nach dem Auftauen tritt eine intraalveolare Hämorragie auf, die die Luft verdrängt und beim nächsten Einfrieren einen größeren Eisball ermöglicht. Im Inneren des entstehenden Eisballs wird im Abstand von 3-5 $\mathrm{mm}$ zu seinem Rand eine zytotoxische Temperatur von $\leq-20^{\circ} \mathrm{C}$ erreicht. Hierdurch wird das Tumorgewebe avital. Die Effektivität nimmt bei Tumoren $>4 \mathrm{~cm}$ signifikant ab [9]. 


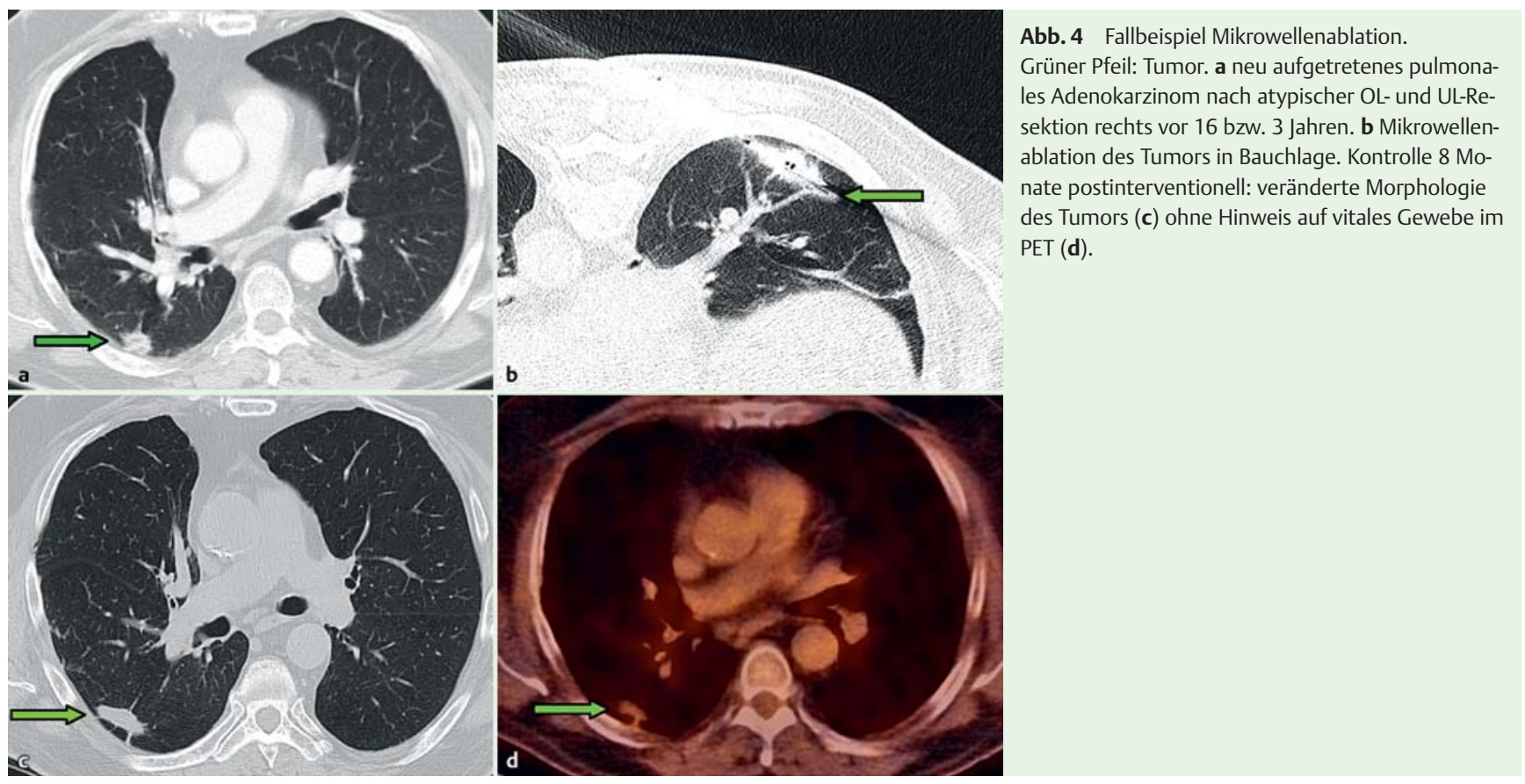

\section{Komplikationen}

In der Veröffentlichung eines Zentrums über 217 Behandlungen werden als Komplikationen der PCA Husten, Hämoptysen, die alle innerhalb einer Woche spontan sistierten, Hypertonie, Pleuraergüsse, Pneumothoraces in 12\% der Fälle und das Auftreten eines Hautemphysems beschrieben. Jeweils ein Fall mit einer Verletzung des Plexus brachialis und des Nervus laryngeus traten auf [9]. In einer anderen Fallserie über 22 Behandlungen trat bei 50\% der Behandlungen ein Pneumothorax auf [10]. Die hohe Pneumothoraxrate kann hierbei durch das dickere Device erklärt werden.

\section{Laserinduzierte Thermotherapie}

Die Machbarkeit, mittels Laser in der Lunge Hitze zu erzeugen und Gewebe zu koagulieren, wurde zunächst im Tiermodell gezeigt [11]. Wie bei der PCA wird auch bei diesem Verfahren eine Sonde in einer modifizierten Seldinger Technik eingebracht. Bei der Behandlung von 64 Patienten mit pulmonalen Metastasen eines extrapulmonalen Primarius durch ein Zentrum traten keine periinterventionellen Todesfälle auf. Bei 38\% der Patienten trat ein Pneumothorax auf und bei 5\% der Patienten war die Behandlung mit einer Drainage erforderlich. Eine definitive Behandlung der pulmonalen Metastasen konnte bei 31 Patienten erzielt werden [12]. Daten zur Behandlung von primären Lungentumoren beim Menschen sind bisher nicht veröffentlicht.

\section{Verfahren mit perkutanem vaskulärem Zugangsweg $\nabla$}

\section{Transpulmonale Chemoembolisation}

Die arterielle Chemoembolisation über einen Katheter (TACE) ist in der Leber bei multinodalem Befall eine Standardtherapie des hepatozellulären Karzinoms [13]. In der Lunge ist das Verfahren in abgewandelter Form anwendbar. Zur sogenannten transpulmonalen Chemoembolisation (TPCE) wird ein Ballonkatheter über eine Femoralvene in die Segmentarterie des tumorbefallenen Lungensegmentes vorgebracht und geblockt. Mittels Applikation von Kontrastmittel lässt sich das Lungengewebe, das von dem Gefäß, in dem der Katheter einliegt, perfundiert wird, darstellen. Gegebenenfalls ist nun eine Um- oder Neupositionierung des Katheters erforderlich, um das tumorbefallene Lungengewebe zu erfassen. Durch Applikation einer zytotoxischen und embolisierenden Suspension, die zum Beispiel aus Mitomycin, Lipiodol und Mikrosphären zusammengesetzt sein kann, wird unter fluoroskopischer Kontrolle eine Stase des Blutflusses im Zielareal erzielt. Die Behandlung kann in 4-wöchentlichem Abstand wiederholt werden. In einer veröffentlichten Fallserie über 52 behandelte Patienten sind nach einer TPCE gelegentlich ein leichter Anstieg der Leukozytenzahl, mildes Fieber und Husten beobachtet worden. In etwa der Hälfte der Patienten mit pulmonalen Metastasen von unterschiedlichen extrapulmonalen Primarii konnte eine "partial response“ oder eine „stable disease“ nach RECISTKriterien erzielt werden [14]. Aussagekräftige Daten zur Anwendung der TPCE bei Bronchialkarzinomen liegen bislang nicht vor. Im Gegensatz zu den oben beschriebenen thermischen Ablationsverfahren verfolgt diese Methode das Ziel einer palliativen lokalen Tumorkontrolle und ist nicht kurativ.

\section{Multiarterielle Chemoperfusion}

Bei dem Verfahren der multiarteriellen Chemoperfusion werden über einen arteriellen Zugang die den Tumor versorgenden Gefäße, die typischerweise aus Ästen der Bronchialarterien abgehen, aufgesucht. Neben Ästen aus den Bronchialarterien können häufig zusätzliche tumorversorgende arterielle Gefäße, die zum Beispiel aus den Interkostalarterien abgehen, detektiert werden. In diese Gefäße wird lokalisiert Chemotherapie, Cisplatin und Gemcitabin oder Doxorubicin und Gemcitabin bei Niereninsuffizienz, appliziert. Hierbei wird eine hohe lokale Dosis der Chemotherapie am Tumor erreicht bei nur geringer systemischer Dosis. Dadurch könnte evtl. auch eine chemotherapeutische Substanz eingesetzt werden, die bei einer systemischen Gabe aufgrund der Dosis nicht tolerabel wäre. In einer veröffentlichen Fallserie über 32 Patienten mit einem NSCLC konnte bei einem Patienten eine vollständige Remission und bei 16 Patienten eine partielle Remission der behandelten Tumoren erzielt werden [15]. 


\begin{tabular}{|c|c|c|c|}
\hline & $\begin{array}{l}\text { Radiofrequenzablation } \\
\text { (RFA) }\end{array}$ & $\begin{array}{l}\text { Mikrowellenablation } \\
\text { (MWA) }\end{array}$ & $\begin{array}{l}\text { Kryoablation } \\
\text { (PCA) }\end{array}$ \\
\hline Tumorgröße & bis $3,5 \mathrm{~cm}$ & bis $3,5 \mathrm{~cm}$ & bis $4 \mathrm{~cm}$ \\
\hline $\begin{array}{l}\text { multifokale Tumoren } \\
\text { behandelbar }\end{array}$ & ja & ja & ja \\
\hline Dauer des Eingriffs & $30-60$ min pro Tumor & $20-45$ min pro Tumor & k. A. \\
\hline Datenlage & $\begin{array}{l}1 \text { einarmige Multicenter- } \\
\text { studie, zahlreiche Fallserien }\end{array}$ & einzelne Fallserien & wenige Fallserien \\
\hline Vorteil & gute Datenlage & $\begin{array}{l}\text { Hinweis auf hohe Effekti- } \\
\text { vität im Randbereich der } \\
\text { Ablationsvolumina }\end{array}$ & $\begin{array}{l}\text { wahrscheinlich risikoärmer } \\
\text { im Bereich mediastinaler } \\
\text { Strukturen }\end{array}$ \\
\hline Nachteil & $\begin{array}{l}\text { abnehmende Effektivität } \\
\text { bei Tumoren }>3,5 \mathrm{~cm}\end{array}$ & $\begin{array}{l}\text { im Vergleich zur RFA größe- } \\
\text { re Sondendurchmesser }\end{array}$ & $\begin{array}{l}\text { hoher Materialaufwand, } \\
\text { zeitaufwendig, im Vergleich } \\
\text { zur RFA größere Sonden- } \\
\text { durchmesser }\end{array}$ \\
\hline
\end{tabular}

Tab. 2 Thermoablative Verfahren in der Behandlung von Malignomen in der Lunge: Radiofrequenz-, Mikrowellenund Kryoablation.

\section{Diskussion}

Interventionell-radiologische Verfahren zur Behandlung lokalisierter pulmonaler Malignome stellen bisher keine Alternative zu einem in kurativer Intention durchgeführten operativen Verfahren dar, sollten jedoch aufgrund des günstigen Risiko-Nutzen-Verhältnisses bei funktionell inoperablen oder eine Operation ablehnenden Patienten neben strahlentherapeutischen Behandlungsmethoden in Erwägung gezogen werden.

Die RFA als das bislang am besten untersuchte Verfahren ist abhängig von der thermischen Leitfähigkeit des zu abladierenden Gewebes. Luft gefüllte, den Tumor umgebende Räume sind einerseits günstig, da sie isolierend die Hitze im Ablationsareal halten und zu einer kürzeren Ablationsdauer führen können. Andererseits bergen sie die Gefahr einer lokalen Tumorprogression im Randbereich des abladierten Tumors. Dieser Effekt spielt bei der Mikrowellenablation theoretisch keine Rolle. Im Vergleich zur RFA scheint die MWA in kürzerer Zeit eine höhere Temperatur innerhalb des Tumors erreichen zu können und ein größeres Ablationsvolumen zu ermöglichen. Diese Vorteile sind bisher jedoch nur theoretisch oder im Tiermodell gezeigt worden [16].

Die Behandlung von Tumoren mittels RFA in der Nähe der Thoraxwand birgt ein erhöhtes Risiko für einen postinterventionellen pleuritischen Schmerz und einen Pneumothorax. In der Nähe von großen Gefäßen oder dem Mediastinum nimmt die Effektivität der RFA aufgrund des hitzeableitenden Effektes größerer Blutgefäße ab. Im Gegensatz zu den hitzeabladierenden Verfahren scheint die PCA in der Nähe mediastinaler Strukturen risikoärmer zu sein [9]. Die Daten zu diesem Verfahren sind spärlich. In der Veröffentlichung einer Fallserie trat innerhalb eines Jahres ein Lokalrezidiv bei $20 \%$ der kryoabladierten Tumoren auf [10]. Die Vor- und Nachteile der thermoablativen Verfahren sind in 0 Tab. 2 dargestellt.

Unter den verschiedenen thermoablativen radiologisch-interventionellen Verfahren besteht derzeit mit der RFA die umfangreichste klinische Erfahrung. Eine kürzlich veröffentlichte Arbeit über 64 Patienten mit NSCLC im Stadium I zeigte nach einer Behandlung mittels limitierter Resektion, RFA oder PCA ein vergleichbares 3-Jahres-Überleben [17]. In der prospektiven, einarmigen Multicenterstudie „RAPTURE Trial“ (Radiofrequency Ablation of Pulmonary Tumours Response Evaluation Trial) [18] wurden pulmonale Rundherde eines NSCLC, aber auch pulmonale Metastasen anderer Karzinome mittels RFA behandelt und für zwei Jahre nachbeobachtet. Nach einem Jahr konnte bei 88\% der Patienten ein Therapieerfolg im Bereich des Zieltumors gezeigt werden. Das Überleben von Patienten mit NSCLC im Stadium I betrug allgemein nach 2 Jahren 75\% und malignomspezifisch $92 \%$. Für die MWA gibt es für die Behandlung des NSCLC nur wenige klinische Daten. In einer Arbeit wurden 82 Lungentumore bei 50 Patienten mittels Mikrowellen behandelt. Bei den Lungentumoren lagen histologisch kleinzellige, nicht-kleinzellige und Metastasen eines extrathorakalen Tumors vor. Eine lokale Kontrolle des Tumors bestand bei $67 \%$ der Patienten nach einem Jahr [19].

Die Sicherheit von Patienten mit implantierten Defibrillator oder Herzschrittmacher wurde in einer Fallserie über 22 Patienten, bei denen neben pumonalen Tumoren auch extrapulmonale Malignome behandelt wurden, überprüft. Im Rahmen der RFA traten Umprogrammierungen des Aggregats auf, bei der MWA traten keine Zwischenfälle auf [20]. Beide Verfahren sollten jedoch bei Patienten mit implantiertem Defibrillator oder Herzschrittmacher allenfalls nach kritischer Risiko- und Nutzenabwägung, unter Beachtung von Sicherheitsmaßnahmen wie der Schrittmacherkontrolle direkt vor und nach einer Intervention sowie ggf. Umprogrammierung in einen V00-Modus durchgeführt werden. Das Hinzuziehen eines kardiologisch erfahrenen Arztes ist in diesen Fällen dringend empfehlenswert.

Chirurgische Resektion von Metastasen eines extrapulmonalen Primarius werden unter der Vorstellung eines Überlebensvorteils durch diese Behandlung durchgeführt, obwohl es bisher keine randomisierten Untersuchungen zum Überleben nach Resektion pulmonaler Metastasen gibt [21]. Unter derselben Rationale erfolgt die lokale Ablation. Die lokale Rezidivfreiheit einer mittels RFA behandelten Metastase scheint unabhängig von ihrer Histologie zu sein [22], sondern ist wesentlich von der Vollständigkeit der Ablation abhängig. 


\section{Fazit}

- Für Patienten mit nicht-kleinzelligem Bronchialkarzinom im Stadium I, II und ggf. IIIa ist eine Lungenteilresektion in kurativer Intention das Verfahren der Wahl bei adäquater kardio-pulmonaler Reserve. Bei lungenfunktionell oder aufgrund ihrer Komorbidität inoperablen Patienten gewinnen radiologisch-interventionelle Verfahren neben strahlentherapeutischen eine zunehmende Bedeutung.

Die RFA oder MWA stellen sowohl bei lokalisierten primären nicht-kleinzelligen Bronchialkarzinomen als auch bei isolierten Metastasen von extrapulmonalen Malignomen potenziell kurative Therapien dar. Die Evaluation ihres Stellenwertes in der Karzinombehandlung gegenüber sonstigen nicht-operativen Behandlungen hinsichtlich des Überlebens und der Lebensqualität erfordert weitere prospektive randomisierte kontrollierte Studien. Eine Kombination dieser Verfahren mit der arteriellen Chemoperfusion kann ggf. die Effizienz steigern. Die risikoarme Durchführbarkeit der interventionell-radiologischen Verfahren wurde bereits gezeigt. Möglicherweise könnte auch eine kombinierte interventionelle lokale Behandlung mit einer adjuvanten systemischen Chemotherapie günstig sein. Zukünftig werden bei den immer älter werdenden Patienten mit zunehmenden Komorbiditäten multimodale Konzepte als interdisziplinäre Behandlungskonzepte unter Einschluss der neueren interventionellen Verfahren eine zunehmende Bedeutung erlangen.

\section{Interessenkonflikt}

Die Autoren geben an, dass kein Interessenkonflikt besteht.

\section{Literatur}

1 Goeckenjan G, Sitter H, Thomas M et al. Deutsche Gesellschaft für Pneumologie und Beatmungsmedizin und die Deutsche Krebsgesellschaft. Prävention, Diagnostik, Therapie und Nachsorge des Lungenkarzinoms. Pneumologie 2010; 64 (Suppl 2): 1-164

2 Bach PB, Cramer LD, Warren JL et al. Racial differences in the treatment of early-stage lung cancer. N Engl J Med 1999; 341: 1198-1205

3 The International Registry of Lung Metastases. Long-term results of lung metastasectomy: prognostic analyses based on 5206 cases. J Thorac Cardiovasc Surg 1997; 113: 37- 49

4 Nordenström BE. Electrochemical treatment of cancer. I: Variable response to anodic and cathodic fields. Am J Clin Oncol 1989; 12: $530-$ 536
5 Gillams AR, Lees WR. Analysis of the factors associated with radiofrequency ablation-induced pneumothorax. Clin Radiol 2007; 62: 639644

6 Hoffmann RT, Jakobs TF, Muacevic A et al. Interventionelle Onkologie bei Lungentumoren. Radiologe 2007; 47: 1109-1116

7 Sano Y, Kanazawa S, Gobara H et al. Feasibility of percutaneous radiofrequency ablation for intrathoracic malignancies: a large single-center experience. Cancer 2007; 109: 1397- 1405

8 Abbas G, Pennathur A, Landreneau RJ, Luketich JD. Radiofrequency and microwave ablation of lung tumors. J Surg Oncol 2009; 100: 645-650

9 Wang H, Littrup PJ, Duan Y et al. Thoracic masses treated with percutaneous cryotherapy: initial experience with more than 200 procedures. Radiology 2005; 235: 289-298

10 Kawamura M, Izumi Y, Tsukada N et al. Percutaneous cryoablation of small pulmonary malignant tumors under computed tomographic guidance with local anesthesia for nonsurgical candidates. J Thorac Cardiovasc Surg 2006; 131: 1007 - 1013

11 RitzJP, Lehmann KS, Mols A et al. Laser-induced thermotherapy for lung tissue - evaluation of two different internally cooled application systems for clinical use. Lasers Med Sci 2008; 23: 195 - 202

12 Rosenberg C, Puls R, Hegenscheid K et al. Laser ablation of metastatic lesions of the lung: long-term outcome. AJR Am J Roentgenol 2009; 192: $785-792$

13 Lencioni $R$. Loco-regional treatment of hepatocellular carcinoma. Hepatology 2010; 52: $762-773$

14 Vogl TJ, Lehnert T, Zangos $S$ et al. Transpulmonary chemoembolization (TPCE) as a treatment for unresectable lung metastases. Eur Radiol 2008; 18: $2449-2455$

15 Nakanishi M, Demura Y, Umeda Yet al. Multi-arterial infusion chemotherapy for non-small cell lung carcinoma - significance of detecting feeding arteries and tumor staining. Lung Cancer 2008; 61: 227-234

16 Pua BB, Thornton RH, Solomon SB. Ablation of pulmonary malignancy: current status. J Vasc Interv Radiol 2010; 21: 223-232

17 Zemlyak A, Moore WH, Bilfinger TV. Comparison of survival after sublobar resections and ablative therapies for stage I non-small cell lung cancer. J Am Coll Surg 2010; 211: 68 - 72

18 Lencioni $R$, Crocetti L, Cioni $R$ et al. Response to radiofrequency ablation of pulmonary tumours: a prospective, intention-to-treat, multicentre clinical trial (the RAPTURE study). Lancet Oncol 2008; 9: 621 -628

19 Wolf FJ, Grand DJ, Machan JT et al. Microwave ablation of lung malignancies: effectiveness, CT findings, and safety in 50 patients. Radiology 2008; 247: $871-879$

20 Skonieczki BD, Wells C, Wasser EJ et al. Radiofrequency and microwave tumor ablation in patients with implanted cardiac devices: Is it safe? Eur J Radiol 2010 [Epub ahead of print]

21 van Raemdonck D, Friedel G. The European Society of Thoracic Surgeons lung metastasectomy project. J Thorac Oncol 2010; 5 (6 Suppl 2): $127-129$

22 Hiraki T, Gobata H, Mimura H et al. Does tumor type affect local control by radiofrequency ablation in the lungs? Eur J Radiol 2010; 74: 136141 